\title{
Critérios para Avaliação do Potencial de lixiviação dos Herbicidas Comercializados No Estado do ParanÁ ${ }^{1}$
}

\author{
Criteria for Evaluation of the Leaching Potential of Herbicides Used in Paraná \\ INOUE, M.H. ${ }^{2,3}$, OLIVEIRA JR., R.S..$^{3,4}$, REGITANO, J.B. ${ }^{5}$, TORMENA, C.A. ${ }^{3,4}$, \\ TORNISIELO, V.L. ${ }^{5}$ e CONSTANTIN, $\mathrm{J}^{4}$
}

\begin{abstract}
RESUMO - Foi realizado um levantamento referente à comercialização de herbicidas no Estado do Paraná, visando quantificar o volume de sua utilização durante o ano de 2000. Em seguida, com base nas propriedades químicas desses herbicidas, estudaram-se critérios teóricos para classificá-los de acordo com o potencial de lixiviação. A análise dos dados do levantamento indicou que o maior volume comercializado ocorreu no quarto trimestre, relacionando-se provavelmente ao aumento da demanda provocada pela safra de verão. Os grupos de mecanismos de ação cujo consumo é mais significativo são, pela ordem, os inibidores da sintese de aminoácidos (36,9\% do total comercializado), os inibidores da fotossintese $(31,3 \%)$, os mimetizadores da auxina (11\%) e os inibidores da divisão celular $(8,8 \%)$. Os herbicidas glyphosate $(4.562,28 \mathrm{t})$, atrazine $(3.075,91 \mathrm{t}), 2,4-\mathrm{D}(1.659,33 \mathrm{t})$ e sulfosate $(631,60 \mathrm{t})$ representam, em conjunto, cerca de $65 \%$ do volume total comercializado no Estado. A classificação quanto ao potencial de lixiviação demonstrou que acifluorfen-sódio, alachlor, atrazine, chlorimuron-ethyl, fomesafen, hexazinone, imazamox, imazapyr, imazaquin, imazethapyr, metolachlor, metribuzin, metsulfuron-methyl, nicosulfuron, picloram, sulfentrazone e tebuthiuron são potencialmente lixiviadores, de acordo com os três critérios teóricos adotados (GUS, CDFA e Cohen).
\end{abstract}

Palavras-chave: contaminação, impacto ambiental, mobilidade, retenção.

\begin{abstract}
A survey was carried out on the commercialization of herbicides in Paraná, to verify their total input in the environment. Based on the chemical properties of these herbicides, theoretical criteria ranking them according to their leaching potential were evaluated. The highest volume of commercialization was found to occur from October to November, probably related to the increased demand caused by the summer crop cycle. The mechanisms of action whose consumption is most expressive are the aminoacid synthesis inhibitors (36.9\% of the total volume), followed by the photosynthesis inhibitors (31.3\%), synthetic auxins (11\%) and mitosis inhibitors (8.8\%). The herbicides glyphosate (4526.28 t), atrazine (3075.91), 2,4-D (1659.33) and sulfosate $(631.60 \mathrm{t})$ account together for around $65 \%$ of the total volume commercialized in the state. Ranking herbicides according to their leaching potential showed that acifluorfen-sodium, alachlor, atrazine, chlorimuron-ethyl, fomesafen, hexazinone, imazamox, imazapyr, imazaquin, imazethapyr, metolachlor, metribuzin, metsulfuron-methyl, nicosulfuron, picloram, sulfentrazone and tebuthiuron are potential leachers according to the three theoretical criteria adopted (GUS, CDFA and Cohen).
\end{abstract}

Key words: contamination, environmental impact, mobility, retention.

1 Recebido para publicação em 20.9.2002 e na forma revisada em 11.8.2003.

Parte da dissertação da primeira autora apresentada para obtenção do título de Mestre em Agronomia.

2 Eng.-Agr., M.S., Universidade Estadual de Maringá - UEM, Professora do Campus de Umuarama-PR. ${ }^{3}$ Bolsista do CNPq.

${ }^{4}$ Professor Adjunto do Departamento de Agronomia da UEM, <rsojunior@uem.br>. ${ }^{5}$ Laboratório de Ecotoxicologia, Centro de Energia Nuclear na Agricultura (CENA-USP), Piracicaba-SP.

Planta Daninha, Viçosa-MG, v.21, n.2, p.313-323, 2003 


\section{INTRODUÇÃO}

Os pesticidas têm sido empregados pelo homem com o objetivo de aumentar a produção mundial de alimentos e reduzir as populações de plantas daninhas, pragas e doenças, elevando o padrão de vida de toda a população mundial (Matthews, 1992). Entretanto, com o aumento do uso desses produtos, aumentaram também as preocupações com a contaminação do solo e da água, bem como com seus efeitos sobre os seres humanos e animais. O processo de lixiviação refere-se ao movimento descendente dos herbicidas na matriz do solo na ou com a água do solo, sendo sua intensidade dependente das características físico-químicas do produto e das características de solo e clima. Segundo Oliveira (2001), para ser lixiviado, o herbicida deve estar na solução do solo ou adsorvido a pequenas partículas, como argilas, ácidos fúlvicos e húmicos de baixo peso molecular, aminoácidos, peptídeos e açúcares, entre outros.

Em 1979, surgiram os primeiros relatos de detecção de nematicidas em aqüiferos subterrâneos de vários Estados norte-americanos, o que despertou a atenção e o interesse para a importância de estudos de impacto ambiental decorrente do uso de pesticidas (Mattos \& Silva, 1999). Existem relatos de muitos outros casos de contaminação de solos, recursos hídricos, animais e seres humanos em vários países, mas pouco tem sido levantado no Brasil. No que diz respeito aos mananciais aquáticos, principalmente os subsuperficiais, a lixiviação desses compostos é uma das principais formas de contaminação.

A lixiviação excessiva contribui, em muitos casos, para que o herbicida atinja e contamine o lençol freático. Nos Estados Unidos e na Europa, atrazine e alachlor estão entre os pesticidas mais freqüentemente detectados em amostras de água do subsolo (Ritter, 1990; Killeen, 1997; Kolpin et al., 1997). Na Georgia (EUA), foi detectado hexazinone tanto na água de escoamento superficial quanto no fluxo descendente de água no solo por vários meses após a aplicação de uma dose de $1,62 \mathrm{~kg} \mathrm{ha}^{-1}$ (Bouchard et al., 1985). Outros herbicidas detectados em água de poço ou lençol subsuperficial são o dicamba (Lym \& Messersmith, 1988; Ritter et al., 1996) e o simazine (Squillace \& Thurman, 1992; Kolpin et al., 1997).

Planta Daninha, Viçosa-MG, v.21, n.2, p.313-323, 2003
O Paraná é, atualmente, o Estado que consome maior volume de herbicidas no Brasil (Sindag, 2002); em conseqüência da agregação de novas áreas produtivas (como o noroeste do Estado), da crescente dificuldade de conseguir mão-de-obra no campo e da grande disponibilidade de produtos químicos, a tendência é de que o consumo de herbicidas continue crescendo de forma consistente nos próximos anos. Embora não existam relatos de detecção de herbicidas em mananciais hídricos no Estado, o fato é que o elevado volume utilizado, aliado à escassez de estudos relacionados ao comportamento dessas substâncias nos solos, evidencia a necessidade de se discutirem parâmetros que possam indicar os herbicidas que eventualmente podem se tornar contaminantes em função de seu potencial de lixiviação.

Para a efetivação de um estudo do potencial de contaminação aquática por herbicidas, é necessário o levantamento de dados básicos referentes ao volume e à sazonalidade de utilização, bem como de informações relacionadas às propriedades químicas dessas moléculas, as quais se encontram dispersas em diferentes fontes da literatura pertinente. Este trabalho objetivou realizar um levantamento para quantificar o volume, a sazonalidade e a distribuição do volume de herbicidas, por princípio ativo, utilizados no Estado do Paraná, no ano de 2000, e analisar critérios teóricos para determinação de seu potencial de lixiviação com base em suas propriedades químicas.

\section{MATERIAL E MÉTODOS}

\section{Quantificação do volume de herbicidas comercializado no Estado do Paraná}

Os dados referentes aos herbicidas utilizados no Estado do Paraná foram obtidos na Secretaria de Estado da Agricultura e do Abastecimento (SEAB), localizada em Curitiba, tendo sido solicitadas informações sobre a quantidade, o produto e a época de comercialização dos herbicidas no ano de 2000. Esses dados são centralizados pela Divisão de Defesa Sanitária Vegetal (DDSV) e provêm das informações coletadas em 18 regiões do Estado do Paraná, que são denominadas pela SEAB de Núcleos Regionais: Núcleo Regional de Apucarana, Campo Mourão, Cascavel, Cornélio 
Procópio, Curitiba, Francisco Beltrão, Guarapuava, Irati, Ivaiporã, Jacarezinho, Londrina, Maringá, Paranaguá, Paranavaí, Pato Branco, Ponta Grossa, Toledo, Umuarama e União da Vitória (Figura 1). Os dados do Núcleo de Paranaguá foram excluídos deste levantamento, por serem praticamente nulos; já o Núcleo de Laranjeiras do Sul foi desmembrado do Núcleo de Guarapuava após a coleta e compilação desses dados.

Os dados coletados nas cópias dos receituários agronômicos enviados aos Núcleos foram agrupados por ingrediente ativo, por grupo químico, por mecanismo de ação, por Núcleo e por trimestre. Espera-se que esses dados reflitam a utilização dos herbicidas em campo e, portanto, forneçam um indicativo da intensidade de uso e do potencial de chegada deles no solo.

\section{Critérios usados para avaliação do potencial de lixiviação}

Para avaliar o risco potencial de lixiviação dos herbicidas no Estado do Paraná, foram utilizados três critérios teóricos (GUS, CDFA e
Cohen), tendo sido usadas as propriedades fisico-químicas dos herbicidas para o cálculo dos indices propostos, compiladas da literatura.

\section{Goundwater Ubiquity Score - GUS}

O indice GUS (indice de vulnerabilidade de águas subterrâneas), proposto por Gustafson (1989), é calculado a partir dos valores de meiavida do composto no solo e do coeficiente de sorção normalizado para o teor de carbono orgânico, não levando em consideração outras propriedades, como solubilidade em água. Este índice é empírico e classifica os compostos de acordo com sua tendência de lixiviação:

$$
\text { GUS }=\log t_{1 / 2}\left(4-\log K_{o c}\right)
$$

em que GUS representa um indice adimensional, $t_{1 / 2}$ representa a meia-vida do herbicida no solo (dias) e $K_{\text {oc }}$ representa o coeficiente de sorção normalizado para o teor de carbono orgânico $\left(\mathrm{L} \mathrm{kg}^{-1}\right)$. Herbicidas com GUS < 1,8 são considerados não-lixiviadores, ao passo que indices superiores a 2,8 representam produtos lixiviadores. Aqueles com valores entre $1,8 \mathrm{e}$ 2,8 são considerados intermediários.

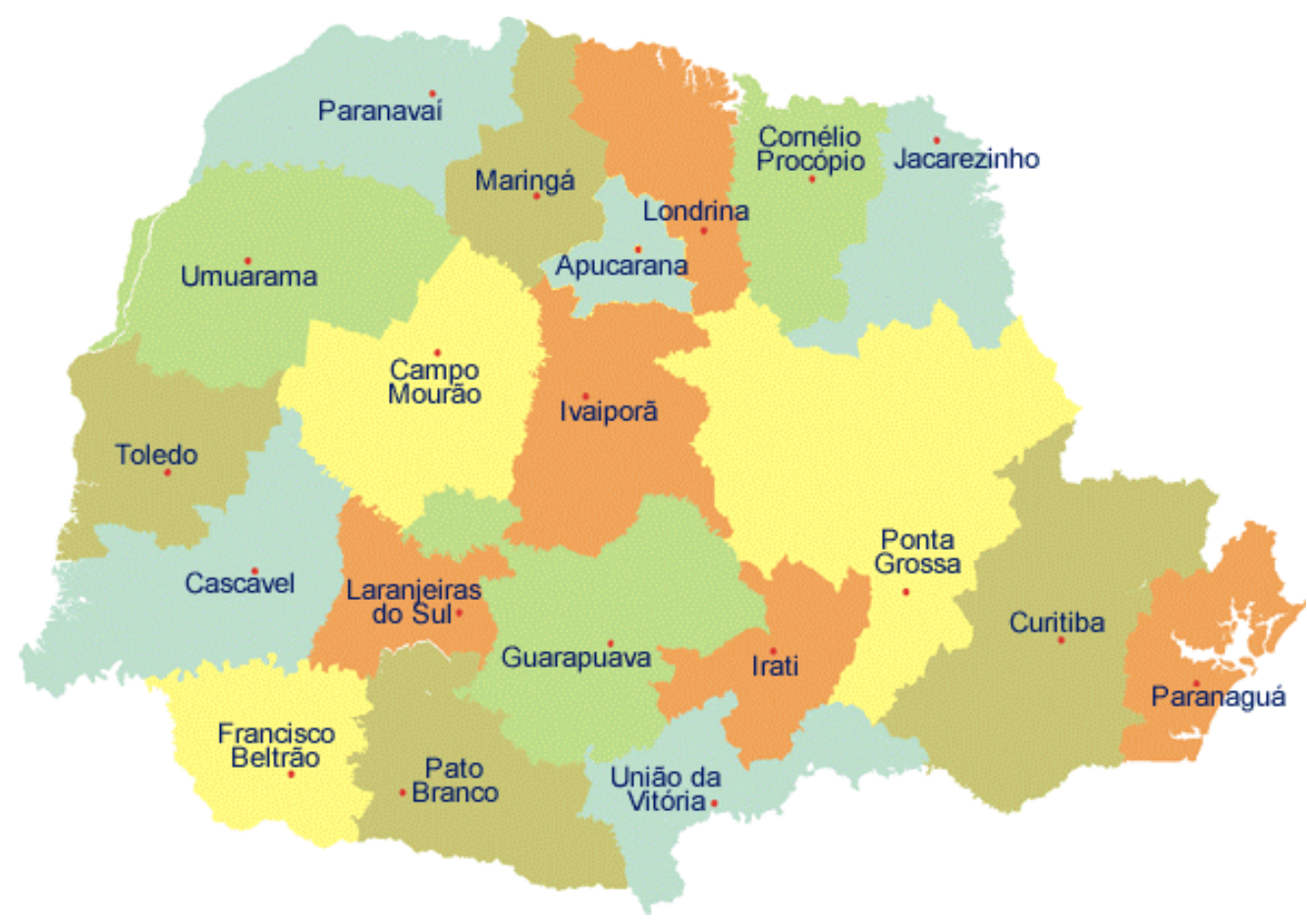

Figura 1 - Distribuição dos Núcleos Regionais da SEAB-PR (2002). 


\section{CDFA}

O critério adotado pelo California Department of Food and Agriculture (CDFA), proposto por Widerson \& Kim (1986), estabelece que herbicidas que apresentem $K_{o c}$ menor que $512 \mathrm{~L} \mathrm{~kg}^{-1}$ e meia-vida superior a 11 dias são classificados como produtos lixiviadores.

\section{Cohen}

Cohen et al. (1984) estabeleceram características do herbicida que podem determinar seu potencial de lixiviação. Herbicidas com $K_{o c}$ menores que $300 \mathrm{~L} \mathrm{~kg}^{-1}$ e valores de meia-vida superiores a 21 dias são considerados lixiviadores, ao passo que aqueles com $K_{o c}$ superiores a $500 \mathrm{~L} \mathrm{~kg}^{-1} \mathrm{e} \mathrm{t}_{1 / 2}$ inferiores a 14 dias representam produtos não-lixiviadores.

\section{RESULTADOS E DISCUSSÃO}

\section{Análise de herbicidas comercializados no Estado do Paraná}

O total de herbicidas comercializado no Estado do Paraná, no ano de 2000, foi de 15.283,2 toneladas de ingrediente ativo (i.a.), o que corresponde, segundo o SINDAG (2002), a $68 \%$ do total de pesticidas comercializados no Estado (Tabela 1).

A Figura 2 apresenta a distribuição da sazonalidade da comercialização. É possivel observar que o período de maior comercialização é o quarto trimestre (7.381,3 t). Esse fato relaciona-se provavelmente ao aumento da demanda provocada pelo início da safra de verão. Dentre as culturas exploradas no verão destacam-se a do milho e a da soja, consideradas as principais do Estado. Essas culturas ocuparam, respectivamente, na safra de 2000/01, 2,79 e 2,78 milhões de hectares no Estado (SEAB-PR, 2001). Os demais trimestres correspondem a 4.049,1 t (terceiro trimestre), 2.286, $8 \mathrm{t}$ (primeiro trimestre) e 1561, $8 \mathrm{t}$ (segundo trimestre). Entretanto, a tendência de picos na comercialização não foi a mesma para todos os Núcleos.

Pela Tabela 1, verifica-se que os Núcleos de Campo Mourão, Cascavel, Ivaiporã, Pato Branco e Ponta Grossa apresentaram maior volume de comercialização no terceiro trimestre (meses de julho, agosto e setembro); no Núcleo de Toledo isso ocorreu no primeiro trimestre (janeiro, fevereiro e março). O elevado volume comercializado no terceiro trimestre pode estar relacionado ao fato de, nestes locais, as datas de plantio terem sido mais precoces do que nos demais, antecipando, conseqüentemente, as vendas para a safra de verão. No Núcleo de Toledo, o elevado volume comercializado no primeiro trimestre deve-se, provavelmente, ao aumento na área de trigo e soja (safrinha) cultivada no ano de 2000. Segundo a SEABPR (2001), no Núcleo de Toledo, na safra de 1999/00 foram cultivados, respectivamente, 11.520 e 800 hectares de trigo e soja (safrinha), ao passo que na safra de 2000/2001 estas culturas ocuparam 122.700 e 4.960 hectares, fazendo com que o maior volume de comercialização de herbicidas deste Núcleo ocorresse no primeiro trimestre. A região norte do Estado foi a que apresentou maior volume de comercialização de herbicidas durante o ano $(6.151,7 \mathrm{t})$, seguida pelas regiões oeste $(3.202,9 \mathrm{t})$, sul $(2.634,1 \mathrm{t})$, centro-oeste $(1.724,35 \mathrm{t})$, sudoeste $(1.088,2 \mathrm{t})$ e noroeste $(481,8 \mathrm{t})$.

Extratificando os princípios ativos segundo sua classificação de herbicidas por grupos de mecanismo de ação (Oliveira Jr., 2001), observa-se que os herbicidas inibidores da sintese de aminoácidos correspondem a 36,9\% do total comercializado e, juntamente com os herbicidas inibidores da fotossintese $(31,3 \%)$, os mimetizadores da auxina $(11,0 \%)$ e os inibidores da divisão celular $(8,8 \%)$, compõem os grupos mais significativos em termos de volume (Figura 3).

De acordo com a distribuição por grupo químico, observa-se pela Tabela 2 que foram comercializadas mais de 5.190 toneladas de i.a. dos herbicidas derivados da glicina; destes, cerca de $88 \%$ referem-se ao consumo de glyphosate. Dessa forma, o grande consumo de herbicidas inibidores da sintese de aminoácidos também está associado basicamente à comercialização do glyphosate, reconhecidamente um dos herbicidas de maior utilização da agricultura mundial, por apresentar largo espectro de espécies controladas, multiutilização e baixo custo, comparado aos demais. Além de ser o herbicida mais utilizado nas operações de manejo em plantio direto, há evidências de que o glyphosate esteja sendo utilizado de 
Tabela 1 - Volume de herbicidas comercializado (em toneladas de ingrediente ativo), distribuído por trimestre, classificado por regiões e núcleos da SEAB-PR, no ano de 2000

\begin{tabular}{|c|c|c|c|c|c|}
\hline \multirow{2}{*}{ Núcleo } & \multicolumn{4}{|c|}{ Trimestre } & \multirow{2}{*}{ Total } \\
\hline & $1^{0}$ & $2^{\underline{0}}$ & $3^{-0}$ & $4^{-}$ & \\
\hline Região norte & 503,15 & 336,70 & 743,55 & $4.568,28$ & $6.151,70$ \\
\hline Apucarana & 30,78 & 56,36 & 40,17 & 93,72 & 221,03 \\
\hline Cornélio Procópio & 134,26 & 110,52 & 207,56 & 541,94 & 994,28 \\
\hline Ivaiporã & 41,14 & 39,70 & 193,68 & 114,70 & 389,22 \\
\hline Jacarezinho & 28,05 & 14,18 & 31,38 & 51,84 & 125,46 \\
\hline Londrina & 77,70 & 17,44 & 120,25 & $3.438,00$ & $3.653,39$ \\
\hline Maringá & 191,22 & 98,50 & 150,51 & 328,08 & 768,32 \\
\hline Região centro-oeste & 257,71 & 301,52 & 684,33 & 476,69 & $1.724,35$ \\
\hline Campo Mourão & 257,71 & 301,52 & 684,33 & 476,69 & $1.724,35$ \\
\hline Região noroeste & 79,41 & 69,74 & 136,35 & 196,34 & 481,84 \\
\hline Paranavaí & 7,97 & 12,17 & 46,52 & 69,31 & 135,97 \\
\hline Umuarama & 71,44 & 57,57 & 89,83 & 127,03 & 345,87 \\
\hline Região oeste & $1.146,06$ & 381,49 & 843,04 & 832,34 & $3.202,94$ \\
\hline Cascavel & 233,66 & 171,04 & 478,11 & 273,65 & $1.156,47$ \\
\hline Toledo & 912,40 & 210,45 & 364,93 & 558,69 & $2.046,47$ \\
\hline Região sudoeste & 103,41 & 69,51 & 533,98 & 381,31 & $1.088,20$ \\
\hline Francisco Beltrão & 51,14 & 38,33 & 173,25 & 182,56 & 445,27 \\
\hline Pato Branco & 52,27 & 31,18 & 360,73 & 198,75 & 642,93 \\
\hline Região sul & 197,13 & 402,86 & $1.107,78$ & 926,33 & $2.634,10$ \\
\hline Curitiba & 49,11 & 102,09 & 150,17 & 160,08 & 461,45 \\
\hline Guarapuava & 27,89 & 37,59 & 29,88 & 106,21 & 201,57 \\
\hline Irati & 6,36 & 6,38 & 26,56 & 39,89 & 79,19 \\
\hline Ponta Grossa & 109,93 & 253,04 & 878,56 & 582,27 & $1.823,80$ \\
\hline União da Vitória & 3,84 & 3,76 & 22,61 & 37,88 & 68,09 \\
\hline Total & $2.286,85$ & $1.561,85$ & $4.049,04$ & $7.381,31$ & $15.283,16$ \\
\hline
\end{tabular}

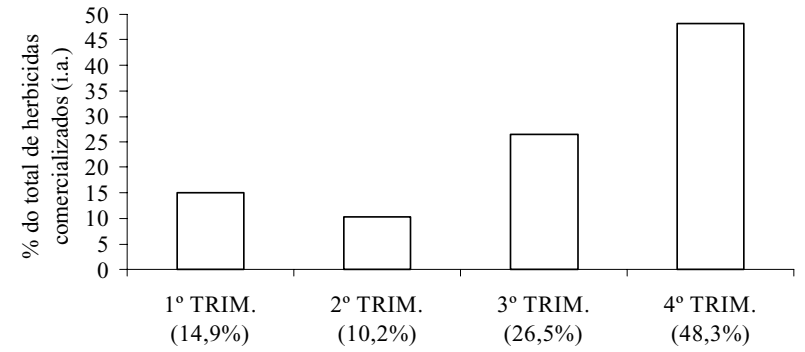

Figura 2 - Sazonalidade de comercialização de herbicidas no Estado do Paraná, no ano de 2000.

outras formas que não aquelas para as quais está registrado - como dessecação pré-colheita da soja, e clandestinamente em pós-emergência, em áreas de soja transgênica. Há, inclusive, relatos de vários casos de cultivo de soja transgênica em áreas no Estado do Paraná, divulgados em jornais da região. Contudo, até o presente momento não há informações precisas a respeito desse assunto, visto que grande quantidade de denúncias ainda está sendo investigada pelos técnicos da SEAB e representantes do Ministério da Agricultura.

A Tabela 3 apresenta os herbicidas mais comercializados no Estado do Paraná em 2000. Glyphosate (4.562,28 t), 2,4-D (1.659,33 t) e sulfosate $(631,60 \mathrm{t})$ representaram juntos cerca de $45 \%$ do total comercializado, uma vez que são muito utilizados na operação de dessecação que antecede a semeadura direta.

O grande volume comercializado de glyphosate, sulfosate e 2,4-D sugere que o aumento da área de plantio direto tem influenciado significativamente o mercado de herbicidas. A área total de plantio direto no Estado do Paraná, que era de apenas 20 mil hectares em 1978, ultrapassou os 3 milhões de hectares 


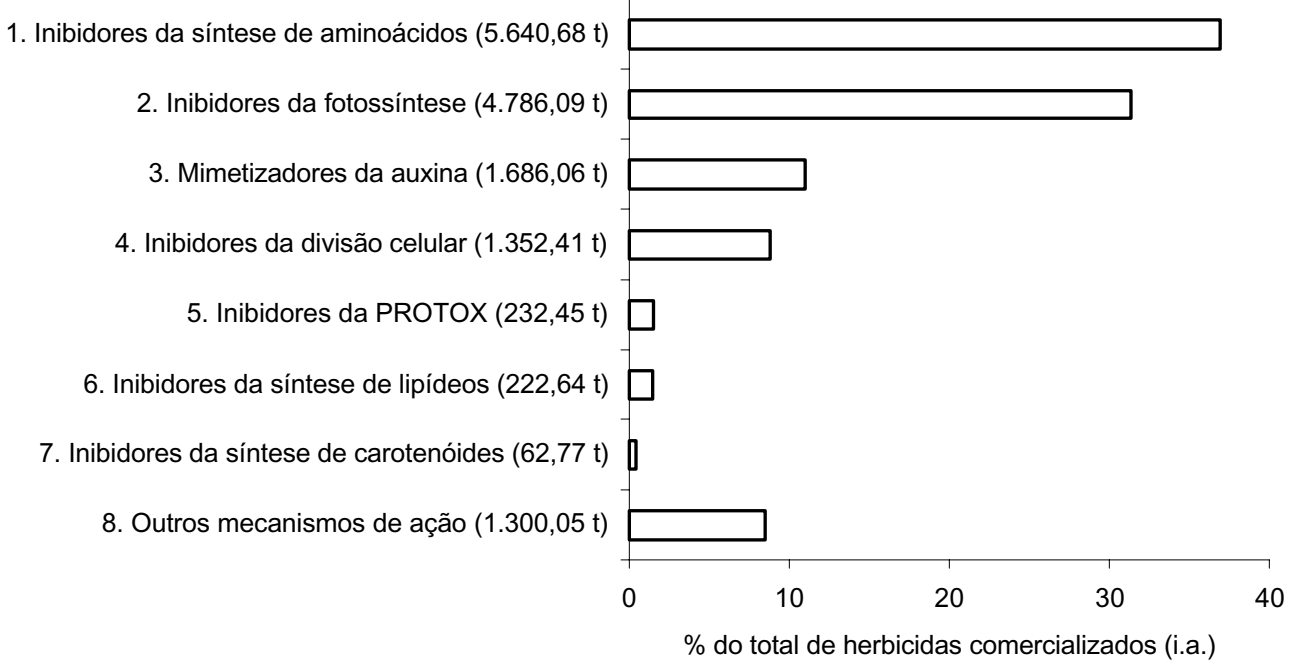

Figura 3 - Resumo do volume de herbicidas comercializado no Paraná, no ano de 2000, extratificado por mecanismo de ação. Os dados referem-se à porcentagem de cada grupo em relação ao total (toneladas de ingrediente ativo) comercializado.

em 1998 (Muzilli, 1999). Constata-se a predominância, principalmente nas regiões norte, sul e oeste do Estado, do plantio direto em áreas cultivadas com milho e soja no verão, seguidas de trigo no inverno, o que vem confirmando as previsões do potencial de expansão do sistema de plantio direto no Paraná.

Apesar de o herbicida paraquat desempenhar basicamente a mesma função do glyphosate, o elevado consumo deste último deve-se provavelmente ao menor preço e também à menor toxicidade, quando comparado ao primeiro. O custo médio de um hectare tratado com glyphosate (1 kg de i.a. ha $\left.{ }^{-1}\right)$ é de $R \$ 22,00$, enquanto o paraquat $(0,3 \mathrm{~kg}$ de i.a. $\mathrm{ha}^{-1}$ ) custa cerca de 2,5 vezes mais, para a mesma área.

Excetuando-se o ghyphosate, o atrazine foi o herbicida mais comercializado no Estado. Esse fato relaciona-se diretamente às explorações agrícolas de milho e apresenta diversas características interessantes do ponto de vista do agricultor, como: ele possui largo espectro de ação e efeito residual, é bastante seletivo às culturas nas quais é utilizado e é também compativel com diversas misturas, tanto formuladas como em tanque. Este herbicida também é registrado para várias outras culturas de menor expressão econômica no Estado, mas que contribuem para expandir seu consumo, como abacaxi, banana, café, citros, seringueira e sorgo.
Tabela 2 - Volume de comercialização de herbicidas no Estado do Paraná, classificado por grupos químicos, no ano de 2000

\begin{tabular}{|l|c|c|}
\hline \multirow{2}{*}{\multicolumn{1}{|c|}{ Grupo químico }} & \multicolumn{2}{|c|}{ Total comercializado } \\
\cline { 2 - 3 } & toneladas de i.a. & $\begin{array}{c}\text { \% em relação ao } \\
\text { total }\end{array}$ \\
\hline Derivados da glicina & $5.193,88$ & 33,98 \\
Triazinas & $3.978,95$ & 26,03 \\
Ácido fenoxicarboxílico & $1.659,33$ & 10,85 \\
Cloroacetanilidas & 684,14 & 4,48 \\
Dinitroanilidas & 634,92 & 4,15 \\
Arsenicais & 436,91 & 2,86 \\
Uréias & 378,69 & 2,48 \\
Bipiridíliuns & 256,53 & 1,68 \\
Imidazolinonas & 205,03 & 1,34 \\
Triazolopirimidinas & 171,02 & 1,11 \\
Difeniléteres & 163,28 & 1,06 \\
Cicloexanodionas & 131,05 & 0,85 \\
Benzothiadiazinonas & 125,51 & 0,82 \\
Ariloxifenoxipropionatos & 91,59 & 0,60 \\
Outros & $1.172,31$ & 7,67 \\
\hline
\end{tabular}

Apesar de ser apenas o $17^{\circ}$ em volume utilizado (Tabela 3), o cloransulam-metil é empregado somente no controle de folhas largas na cultura da soja, em baixas doses (cerca de 40 g de i.a. ha-1), sendo a quantidade comercializada $(88,7$ t) supostamente suficiente para tratar mais de 2,5 milhões de hectares, ou seja, cerca de $90 \%$ do total de área da cultura da soja no Estado. Um outro herbicida registrado 
somente para a cultura da soja é o chlorimuronethyl, que, aplicado tanto isoladamente quanto em mistura, permite controlar infestantes problemáticas no Estado. Embora este herbicida não esteja entre os mais comercializados em volume, devido à sua baixa dosagem (apenas $20 \mathrm{~g} \mathrm{ha}^{-1}$ ), a quantidade comercializada é suficiente para mais de 2,2 milhões de hectares. Ao levar em consideração o somatório de áreas de soja que estes dois herbicidas poderiam ter tratado (4,7 milhões de hectares) e o quanto é cultivado no Estado (2,8 milhões de hectares), é possivel evidenciar que, embora estes herbicidas tenham sido comercializados no Paraná, parte destes produtos pode estar sendo utilizada em outros Estados ou países. Outro ponto é que pode haver mistura de produtos em uma mesma área ou necessidade de aplicações seqüenciais de herbicidas diferentes.

Os herbicidas inibidores da sintese de lipídios, dos grupos das ciclohexanodionas e ariloxifenoxipropionatos, representaram mais de 220 toneladas de i.a., sendo a importância destes herbicidas a de se concentrar no controle de gramíneas em pós-emergência.

Tabela 3 - Volume de comercialização de herbicidas, classificados por princípio ativo, mais comercializados no Estado do Paraná, no ano de 2000

\begin{tabular}{|l|c|c|}
\hline \multirow{2}{*}{ Nome comum } & \multicolumn{2}{|c|}{ Total comercializado } \\
\cline { 2 - 3 } & toneladas de i.a. & $\begin{array}{c}\text { \% em relação ao } \\
\text { total }\end{array}$ \\
\hline Glyphosate & $4.562,28$ & 29,85 \\
Atrazine & $3.075,91$ & 20,12 \\
2,4-D & $1.659,33$ & 10,85 \\
Sulfosate & 631,60 & 4,13 \\
Simazine & 568,38 & 3,72 \\
Trifluralin & 550,91 & 3,60 \\
MSMA & 436,92 & 2,58 \\
Metolachlor & 387,99 & 2,54 \\
Diuron & 363,23 & 2,37 \\
Ametryne & 300,35 & 1,96 \\
Paraquat & 235,51 & 1,54 \\
Alachlor & 197,87 & 1,29 \\
Bentazon & 125,51 & 0,82 \\
Imazaquin & 119,31 & 0,78 \\
Acetochlor & 98,28 & 0,64 \\
Cloransulam-metil & 88,77 & 0,58 \\
Fomesafen & 86,23 & 0,57 \\
Clethodim & 85,16 & 0,56 \\
Pendimethalin & 84,01 & 0,55 \\
Outros & $1.625,62$ & 10,63 \\
\hline
\end{tabular}

\section{Potencial de lixiviação dos herbicidas comercializados no Estado do Paraná}

A Tabela 4 apresenta a avaliação do potencial de lixiviação dos herbicidas comercializados no Estado do Paraná, segundo os critérios teóricos GUS (Gustafson, 1989), CDFA (Widerson \& Kim, 1986) e Cohen (Cohen et al., 1984). O ranqueamento demonstrou que acifluorfen-sódio, alachor, atrazine, chlorimuron-ethyl, fomesafen, hexazinone, imazamox, imazapyr, imazaquin, imazethapyr, metolachlor, metribuzin, metsulfuron-methyl, nicosulfuron, picloram, sulfentrazone e tebuthiuron são potencialmente lixiviadores, de acordo com os três critérios utilizados. Esse fato reveste-se de acentuada importância, visto que a lixiviação por meio do perfil do solo tem sido identificada como a maior causa da ocorrência de pesticidas no lençol freático (Flury, 1996). Sabe-se, porém, que o risco de contaminação do lençol freático subterrâneo depende de diversas propriedades físicas e químicas do herbicida, do tipo de solo (teor de matéria orgânica, composição física e química, $\mathrm{pH}$ ), da cobertura morta e das condições climáticas (Milanova \& Grigorov, 1996). Além destes, acetochlor, ametryne, bentazon, clomazone, dicamba, diclofop e simazine foram considerados lixiviadores em dois dos três critérios utilizados ("2 L").

Dentre os herbicidas envolvidos neste estudo, alachlor, atrazine e simazine são os mais freqüentemente detectados em amostras de água do subsolo (Pereira \& Rostad, 1990; Thurman et al., 1991, 1992, 1996; Squillace e Thurman, 1992; Buhler et al., 1993; Pereira \& Hostettler, 1993; Domagalsky, 1996; Domagalsky et al., 1997; Kolpin et al., 1997; Ma \& Spalding, 1997), o que gerou inclusive a obrigatoriedade da referência acerca do potencial que esses herbicidas possuem de contaminação de águas subterrâneas em rótulos de embalagens comerciais nos EUA. No caso particular de simazine, este não foi classificado como lixiviador, segundo o critério GUS, devido ao valor de 22 dias de meia-vida assumido (obtido em solos de Campinas-SP por Blanco et al., 1997) para o cálculo de índice. No entanto, se fosse assumido um valor de 28 dias, como o obtido em trabalhos conduzidos na Austrália (Kookana et al., 1995), ou o valor médio de 60 dias proposto por Ahrens (1994), como nos critérios de CDFA e Cohen, ele seria classificado também como lixiviador.

Planta Daninha, Viçosa-MG, v.21, n.2, p.313-323, 2003 
Tabela 4 - Classificação do potencial de lixiviação dos herbicidas comercializados no Estado do Paraná no ano de 2000, segundo os critérios de GUS, CDFA e Cohen. $\mathrm{L}=$ lixiviador, $\mathrm{IN}$ = intermediário e $\mathrm{NL}=$ não-lixiviador

\begin{tabular}{|c|c|c|c|c|c|c|}
\hline \multirow{2}{*}{ Nome comum } & \multirow{2}{*}{$\begin{array}{c}\mathrm{K}_{\mathrm{oc}} \\
\left(\mathrm{mL} \mathrm{g}^{-1}\right)\end{array}$} & \multirow{2}{*}{$\begin{array}{c}\mathrm{t}_{1 / 2} \\
\text { (dias) }\end{array}$} & \multirow{2}{*}{ Fonte dos dados } & \multicolumn{3}{|c|}{ Critério } \\
\hline & & & & GUS & CDFA & Cohen \\
\hline $2,4-\mathrm{D}$ & 60 & 10 & Wauchope et al., 1992 & IN & - & - \\
\hline Acetochlor & $55^{\underline{z}+1}$ & 20 & Ahrens, 1994; & $\mathrm{L}$ & L & - \\
\hline Acifluorfen-sódio & 113 & $44 *$ & Wauchope et al., 1992; Ahrens, 1994 & $\mathrm{~L}$ & $\mathrm{~L}$ & $\mathrm{~L}$ \\
\hline Alachlor & 103 & 80 & Wauchope et al., 1992; Oliveira Jr. et al., 2001 & $\mathrm{~L}$ & $\mathrm{~L}$ & $\mathrm{~L}$ \\
\hline Ametryne & 300 & 60 & Wauchope et al., 1992 & IN & $\mathrm{L}$ & $\mathrm{L}$ \\
\hline Amônio-glufosinato & 100 & 7 & Wauchope et al., 1992 & NL" & - & - \\
\hline Atrazine & 165 & 60 & Wauchope et al., 1992; Oliveira Jr. et al., 2001 & L & L & $\mathrm{L}$ \\
\hline Bentazon & 34 & 20 & Wauchope et al., 1992 & L & $\mathrm{L}$ & - \\
\hline Butroxydim ${ }^{19}$ & $300^{-2 / 1}$ & - & Syngenta & - & - & - \\
\hline Clethodim & $16,5^{-21 "}$ & 3 & Ahrens, 1994 & NL" & - & - \\
\hline Clomazone & 300 & 24 & Wauchope et al., 1992 & IN & L & L \\
\hline Chlorimuron ethyl & 110 & 40 & Wauchope et al., 1992 & L & $\mathrm{L}$ & $\mathrm{L}$ \\
\hline Cloransulam-metil & $485^{*}$ & $9 *$ & Hatzios, 1998 & NL & - & - \\
\hline Cyanazine & $190^{2 / 7}$ & 14 & Wauchope et al., 1992 & IN & $\mathrm{L}$ & - \\
\hline Dicamba & 2 & 14 & Wauchope et al., 1992; Oliveira Jr. et al., 2001 & L & L & - \\
\hline Diclofop-methyl & 251 & 30 & Wauchope et al., 1992; Ahrens, 1994 & "IN & L & $\mathrm{L}$ \\
\hline Diclosulam ${ }^{-}$ & - n & $49 *$ & Rodrigues \& Almeida, 1998 & - & - & - \\
\hline Diquat & 1000000 & 1000 & Wauchope et al., 1992 & NL" & - & - \\
\hline Diuron & 480 & 90 & Wauchope et al., 1992 & "IN & $\mathrm{L}$ & - \\
\hline EPTC & 200 & 6 & Wauchope et al., 1992 & NL" & - & - \\
\hline Fenoxaprop-p-ethyl & 9490 & 180 & Wauchope et al., 1992 & NL & - & - \\
\hline Fluazifop-p-butil & 5700 & 15 & Wauchope et al., 1992 & NL & - & - \\
\hline Flumetsulam & $356^{*}$ & $60 *$ & Ahrens, 1994 & IN & $\mathrm{L}$ & - \\
\hline Flumiclorac-pentil ${ }^{1 /}$ & - & $3,5^{*}$ & 'Ahrens, 1994 & - & - & - \\
\hline Flumioxazin ${ }^{1}$ & - n & 21,9 & Rodrigues \& Almeida, 1998 & 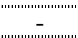 & - & - \\
\hline Fluroxipir-MHE ${ }^{1 /{ }^{\prime}}$ & - & $48,5 *$ & Rodrigues \& Almeida, 1998 & - & - & - \\
\hline Fomesafen & 60 & 100 & Wauchope et al., 1992; Ahrens, 1994 & L & L & L" \\
\hline Glyphosate & 24000 & 47 & Wauchope et al., 1992 & NL" & - & - \\
\hline Halosulfuron & 93,5 & 16 & Rodrigues \& Almeida, 1998 & "IN & $\mathrm{L}$ & - \\
\hline Hexazinone & 34 & 90 & Wauchope et al., 1992; Oliveira Jr. et al., 2001 & $\mathrm{~L}$ & $\mathrm{~L}$ & $\mathrm{~L}$ \\
\hline Imazamox & 5,36 & $25^{*}$ & Hatzios, 1998 & $\mathrm{~L}$ & $\mathrm{~L}$ & $\mathrm{~L}$ \\
\hline Imazapic ${ }^{1 / 2}$ & - n l- & $>180$ & Rodrigues \& Almeida, 1998 & - & - & - \\
\hline Imazapyr & 100 & $90 *$ & Hornsby et al., 1995 & L & L & $\mathrm{L}$ \\
\hline Imazaquin & 20 & 60 & Wauchope et al., 1992 & L & L & $\mathrm{L}$ \\
\hline Imazethapyr & 22 & $75 *$ & Ahrens, 1994; Oliveira Jr. et al., 2001 & L & L & L \\
\hline Ioxynil-octanoato ${ }^{\underline{T}}$ & - & - & - & - & - & - \\
\hline Isoxaflutole $\mathrm{I}^{\mathrm{I} /}$ & $196^{\frac{27}{2}}$ & - & IBAMA, $2001^{4 \prime}$ & - & - & - \\
\hline Lactofen & 10000 & 3 & Wauchope et al., 1992 & NL & - & NL \\
\hline Linuron & 400 & 60 & Wauchope et al., 1992 & IN & L & - \\
\hline Metolachlor & 200 & $195 *$ & Wauchope et al., 1992 & L & L & $\mathrm{L}$ \\
\hline Metribuzin & 60 & $45 *$ & Wauchope et al., 1992 & L & L & L \\
\hline Metsulfuron-methyl & 12 & 30 & Wauchope et al., 1992; Oliveira Jr. et al., 2001 & L & L & $\mathrm{L}$ \\
\hline MSMA & 7000 & 185 & Ahrens, 1994 & NL" & - & - \\
\hline Nicosulfuron & 20 & 21 & Augustijn-Beckers et al., 1994; Oliveira Jr. et al., 2001 & L & L & $\mathrm{L}$ \\
\hline Oxadiazon & 3200 & 60 & Wauchope et al., 1992 & NL & - & - \\
\hline Oxasulfuron $^{\mathrm{I}}$ & - & - & - & - & - & - \\
\hline Oxyfluorfen & 100000 & $35^{*}$ & Wauchope et al., 1992 & NL" & - & - \\
\hline Paraquat & 1000000 & 1000 & Wauchope et al., 1992 & NL" & - & - \\
\hline Pendimethalin & 17200 & 44 & Ahrens, 1994 & NL" & - & - \\
\hline Picloram & $88,5^{*}$ & 90 & Wauchope et al., 1992 & L & L & $\mathrm{L}$ \\
\hline Prometryne & 400 & 60 & Wauchope et al., 1992 & IN & L & - \\
\hline Propanil & 149 & 1 & Wauchope et al., 1992 & NL" & - & - \\
\hline Propaquizafop & $236^{-2 r}$ & - & IBAMA, $2001^{4}$ & - & - & - \\
\hline Pyrithiobac-sodium ${ }^{17}$ & - & 62 & Webster \& Shaw, 1997 & - & - & - \\
\hline Quizalofop-p-ethyl & 510 & 60 & Wauchope et al., 1992 & IN & $\mathrm{L}$ & - \\
\hline Sethoxydim & 100 & 5 & Wauchope et al., 1992 & NL & - & - \\
\hline Simazine & $<97$ & 22 & Blanco et al., 1997; Oliveira Jr. et al., 2001 & IN & $\mathrm{L}$ & $\mathrm{L}$ \\
\hline Sulfentrazone & (3) & $250^{*}$ & Grey et al., 1997; Hatzios, 1998 & L't & $\mathrm{L}$ & $\mathrm{L}$ \\
\hline Sulfosate & $57000^{2 !}$ & - & Syngenta, $2002^{3^{3}}$ & 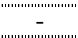 & - & - \\
\hline Tebuthiuron & 80 & $585^{*}$ & Wauchope et al., 1992 & L & L & L \\
\hline Tepraloxydim & - n & - & - & 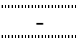 & - & - \\
\hline Thiobencab & 1698 & $60 *$ & Ahrens, 1994 & NL" & - & - \\
\hline Trifluralin & 7000 & 45 & Ahrens, 1994 & NL & - & - \\
\hline
\end{tabular}

* Valor médio extraído da(s) fonte(s).

1/ Valores de $\mathrm{K}_{\mathrm{Oc}}, \mathrm{K}_{\mathrm{d}}$ e/ou $\mathrm{t}_{1 / 2}$ 